\title{
COMPLETENESS OF COMPACT LORENTZ MANIFOLDS ADMITTING A TIMELIKE CONFORMAL KILLING VECTOR FIELD
}

\author{
ALFONSO ROMERO AND MIGUEL SÁNCHEZ
}

(Communicated by Christopher B. Croke)

\begin{abstract}
It is proved that every compact Lorentz manifold admitting a timelike conformal Killing vector field is geodesically complete. So, a recent result by Kamishima in J. Differential Geometry [37 (1993), 569-601] is widely extended.
\end{abstract}

Recently, it has been proved in [2] that a compact Lorentz manifold of constant curvature admitting a timelike Killing vector field is (geodesically) complete. It is natural to think about the importance of the assumption on the curvature in this result. Moreover, we could ask ourselves if there is a more general condition than the existence of a timelike Killing vector field. The answers to these questions are given in the following

Theorem. Let $(M, g)$ be a compact Lorentz manifold which admits a timelike conformal Killing vector field $K$. Then $(M, g)$ is geodesically complete.

Proof. We are going to see that any geodesic $\gamma:[0, b[\rightarrow M, 0<b<\infty$, is extendible beyond $b$. It suffices to show that the vector field $\gamma^{\prime}$ remains in a compact subset of the tangent bundle. As $g\left(\gamma^{\prime}, \gamma^{\prime}\right)$ is a constant $C$, it is enough to see that the projection onto the subbundle $\operatorname{Span}\{K\} \operatorname{maps} \gamma^{\prime}$ into a compact subset of $\operatorname{Span}\{K\}$ (here $\operatorname{Span}\{K\}$ denotes the line bundle $\left.\left\{\lambda \cdot K_{p} \mid \lambda \in \mathbb{R}, p \in M\right\}\right)$. As $K$ is timelike, the compactness of $M$ implies that Inf $|g(K, K)|>0$. Thus, we have only to check that $g\left(K, \gamma^{\prime}\right)$ is bounded. Taking into account the fact that $L_{K} g=\sigma \cdot g$ for some function $\sigma$, we obtain that

$$
\frac{d}{d t} g\left(K, \gamma^{\prime}\right)=\frac{1}{2} C \cdot \sigma \circ \gamma
$$

so $(d / d t) g\left(K, \gamma^{\prime}\right)$ and, as a consequence $g\left(K, \gamma^{\prime}\right)$ is bounded on $[0, b[$.

Received by the editors October 20, 1993 and, in revised form, January 25, 1994.

1991 Mathematics Subject Classification. Primary 53C50, 53C22.

Key words and phrases. Compact Lorentz manifolds, geodesic completeness, conformal Killing vector fields, timelike vector fields.

Research partially supported by a DGICYT Grant No. PB91-0731.

(c) 1995 American Mathematical Society 
Remarks. (1) The assumptions of this result can be weakened. An almost similar proof works if we are given a compact semi-Riemannian manifold with so many timelike (resp. spacelike) pointwise independent conformal Killing vector fields as the index (resp. the coindex) of the semi-Riemannian metric.

(2) Under the assumptions of our Theorem, if $(M, g)$ is also of constant curvature $k$, then $k \leq 0$. This fact follows directly from the completeness of $g$ and Theorem 11.1.7 in [4] (giving an alternative proof to one of the assertions in Theorem $\mathrm{A}$ in [2]).

(3) It is a main problem concerning semi-Riemannian manifolds whether compactness of a semi-Riemannian manifold with any extra assumption implies (geodesic) completeness. It should be emphasized that simply the existence of a nontrivial Killing vector field does not imply the completeness of a compact Lorentz manifold (recall Clifton-Pohl torus). Even more, completeness is not obtained if there exists a causal Killing vector field. As a counterexample, consider the following Lorentz metric on $\mathbb{R}^{2}$

$$
g=\left(1-\cos 2 \pi x_{1}\right)\left(d x_{1}^{2}-d x_{2}^{2}\right)+\left(1+\cos 2 \pi x_{1}\right)\left(d x_{1} \otimes d x_{2}+d x_{2} \otimes d x_{1}\right) .
$$

Although $\partial / \partial x_{2}$ is a causal Killing vector field of $g$, this metric is incomplete and yields, in a natural way, a Lorentz torus as required (see [3] for details).

(4) The next results allow us to obtain completeness of a compact Lorentz manifold, with extra assumptions involving curvature:

(A) If a compact Lorentz manifold has zero curvature, then it is geodesically complete [1].

(B) Let $M$ be a Lorentz 3-manifold of constant negative curvature. Suppose that the universal covering space $\bar{M}$ of $M$ admits a nontrivial complete Killing vector field and the developing map is injective. If $M$ is compact, then $M$ is geodesically complete ([2], Theorem $\mathrm{D}$, see also Theorem $\mathrm{E})$.

As a nice consequence of our Theorem combined with the result (A) we have the corollary:

(C) A Lorentz $n$ ( $\geq 2$ )-torus which is globally conformal to a flat Lorentz torus is geodesically complete.

(5) A natural question is to study which manifolds lie under the assumptions in our Theorem. The referee pointed out to us the following discussion. Let $(M, g)$ be a compact Lorentz manifold with a timelike conformal Killing vector field $K$. As $g(K, K) \neq 0$, a Riemannian metric $g^{\prime}$ is defined to be

$$
g^{\prime}(A, B)=-\left(g(A, B)-\frac{2 g(A, K) g(B, K)}{g(K, K)}\right) / g(K, K) \text {. }
$$

It is noted that the one-parameter group generated by $K$ preserves the Riemannian metric $g^{\prime}$. Since the isometry group Iso $\left(M, g^{\prime}\right)$ is compact, there is a circle $S^{1}$ whose Killing vector field $K^{\prime}$ is sufficiently close to $K$. Thus $K^{\prime}$ is timelike with respect to $g$. So $M$ is a Lorentz manifold for which $S^{1}$ acts on $M$ without fixed points. In dimension three, topologically $M$ is a Seifert manifold. When $S^{1}$ happens to act freely, $M$ is a principal circle bundle over a compact Riemannian manifold $N$ whose Riemannian metric is induced by $g$. So the curvature of $g$ controls the topology of $N$ (compare with [2]). Therefore, it is unavoidable for the hypothesis to restrict those Lorentz manifolds into a special class. 


\section{ACKNOWLEDGMENT}

The authors would like to express their thanks to Y. Carrière for bringing to their attention the reference [2], and to the referee for his valuable suggestions.

\section{REFERENCES}

1. Y. Carrière, Autour de la conjecture de L. Markus sur les variétés affines, Invent. Math. 95 (1989), 615-628.

2. Y. Kamishima, Completeness of Lorentz manifolds of constant curvature admitting Killing vector fields, J. Differential Geom. 37 (1993), 569-601.

3. A. Romero and M. Sánchez, Incomplete Lorentzian tori with a Killing vector field, preprint, Univ. Granada, 1993.

4. J.A. Wolf, Spaces of constant curvature, McGraw-Hill, New York, 1967.

Departamento Geometria y Topología, Facultad Ciencias, Universidad de Granada, 18071 Granada, Spain

E-mail address: aromero@ugr.es

E-mail address: msanchez@ugr.es 